\title{
Dynamical response function in sodium studied by inelastic x-ray scattering spectroscopy
}

\author{
Simo Huotari, ${ }^{1,2,{ }^{*}}$ Marco Cazzaniga, ${ }^{3,4}$ Hans-Christian Weissker, ${ }^{4,5,6}$ Tuomas Pylkkänen, ${ }^{1,2}$ Harald Müller, ${ }^{2}$ \\ Lucia Reining, ${ }^{4,5}$ Giovanni Onida, ${ }^{3,4}$ and Giulio Monaco ${ }^{2}$ \\ ${ }^{1}$ Department of Physics, University of Helsinki, P. O. Box 64, FI-00014 Helsinki, Finland \\ ${ }^{2}$ European Synchrotron Radiation Facility, B. P. 220, F-38043 Grenoble Cedex, France \\ ${ }^{3}$ Università degli Studi di Milano, Dipartimento di Fisica, via Celoria 16, 20133 Milano, Italy \\ ${ }^{4}$ European Theoretical Spectroscopy Facility (ETSF) \\ ${ }^{5}$ Laboratoire des Solides Irradiés, UMR 7642, CNRS-CEA/DSM, Ecole Polytechnique, Palaiseau, France \\ ${ }^{6}$ CINaM, CNRS, Campus de Luminy, Case 913, 13288 Marseille Cedex 9, France ${ }^{\dagger}$ \\ (Received 15 November 2010; revised manuscript received 9 May 2011; published 5 August 2011)
}

\begin{abstract}
We present the dynamic structure factor $S(Q, \omega)$ of $\mathrm{Na}$ valence electrons in the range of momentum transfer $0.5 k_{F}<Q<2.4 k_{F}$ and energy transfer $3 \mathrm{eV}<\omega<30 \mathrm{eV}$ determined by inelastic x-ray scattering spectroscopy. In this range, we observe how the collective plasmon excitations decay into the single-particle excitation continuum. We compare the results to calculations using time-dependent density-functional theory with different approximations. The failure of both random-phase approximation and time-dependent local-density approximation (TDLDA) is shown to become important at $k_{F}<Q<2.4 k_{F}$, while TDLDA with an additional inclusion of quasiparticle lifetime effects reproduces the experimental spectra well. The experimental valenceelectron response reaches the single-particle spectrum surprisingly early, at $Q \approx 1.5 k_{F}$. This is manifested both in the spectral shape and the peak dispersion. The experimental spectra are nearly free of any fine structure, confirming that the peak-shoulder structure observed in many other materials is due to band-structure effects, which turn out to be negligible in Na.
\end{abstract}

DOI: 10.1103/PhysRevB.84.075108

PACS number(s): 78.70.Ck, 71.45.Gm

\section{INTRODUCTION}

The response of a material to an electromagnetic field is described by its dielectric function $\varepsilon(\mathbf{Q}, \omega){ }^{1}$ which is a function of momentum $\mathbf{Q}$ and frequency $\omega$ or, equivalently, of their Fourier representations, space and time. The response to visible light corresponds to the limiting case $Q=|\mathbf{Q}| \rightarrow 0$. For finite $Q$, which could probe short-distance correlations, the response can be studied, e.g., via electron energy-loss and inelastic X-ray scattering spectroscopies [EELS (Refs. 2 and 3) and IXS, (Refs. 4 and 5) respectively]. Two distinct limiting regimes with respect to the so-called critical plasmon cut-off vector $Q_{c}$ can be observed by these techniques: (i) $Q<$ $Q_{c}$, where collective plasmon modes are responsible for the dissipation, and (ii) $Q>Q_{c}$ where the single-particle response is probed, leading to the so-called Compton regime ${ }^{6}$ at very large $Q$ where, within the impulse approximation, the groundstate momentum density of the electrons can be observed. ${ }^{7}$

The homogeneous electron gas (HEG), also known as jellium, is the canonical testing ground for many-body theories. The HEG approximation is often assumed to be roughly valid to explain the dielectric response of certain solids, especially the alkali metals. Typical $Q_{c}$ values fall in the range of 0.1$1.0 a_{0}^{-1}$ for condensed matter systems, $a_{0}$ being the Bohr radius. The accessible $Q$ range is typically $Q \lesssim 0.5 a_{0}^{-1}$ for EELS and $Q \gtrsim 0.5 a_{0}^{-1}$ for IXS, although there is a certain overlap-EELS studies have also been performed at rather high $Q$ up to $0.9 a_{0}^{-1}$ (Ref. 8) and, conversely, IXS measurements at rather small $Q$ down to $0.1 a_{0}^{-1}$ (Refs. 9-11). Nevertheless, both experimental methods may become difficult outside their "natural" range.

The IXS technique has developed rapidly in recent years and several experimental facilities have been installed around the world. ${ }^{12-16}$ Developments in the instrumentation ${ }^{17-19}$ have improved the resolving power and efficiency up to a level where the technique has become a standard tool in condensedmatter physics.

The first IXS measurements were performed with conventional x-ray sources. ${ }^{20-22}$ The results showed for all studied materials (beryllium, aluminum, and graphite) deviations from the random-phase-approximation (RPA) prediction for the HEG; most importantly, a redshift of the spectral weight, a more symmetric lineshape, and a double-peak (or peakand-shoulder) structure. Due to the similarity of the results for $\mathrm{Be}, \mathrm{Al}$, and graphite, such deviations were interpreted as reflecting universal properties of the HEG beyond the RPA, i.e., due to strong correlation at short distances, not treated properly in the RPA. This suggestion launched an enormous amount of theoretical work on the electron-electron interaction effects on the dielectric function. Several works explained the double-peak or peak-shoulder structure as a universal HEG response, ${ }^{23-33}$ while some did not. ${ }^{34-42}$ An interesting calculation for an asymptotically exact dynamic structure factor for a specific $r_{s} \approx 3.5$ (between $\mathrm{Na}$ and $\mathrm{Li}$ ) was done by Hong and Lee, giving as a result an $S(Q, \omega)$ without any fine structure. ${ }^{41}$ On the other hand, a recent work by Takada and Yasuhara ${ }^{43}$ discovered that a low-energy shoulder should appear in the HEG $S(Q, \omega)$ due to excitonic effects.

From the experimental side, an important contribution was given by synchrotron-based experiments on lithium, ${ }^{44,45}$ beryllium, ${ }^{46,47}$, and aluminum ${ }^{48}$ where the fine structure of the dynamic structure factor was shown to be strongly crystalorientation dependent. The spectral structures were explained by excitation gaps, caused by the absence of unoccupied states of certain energies and visible in the density of empty states (DOS) of the material. For instance, in aluminum ${ }^{48}$ and potassium $^{49}$ such gaps in the DOS above $\varepsilon_{F}$ were shown to be predominantly due to states with $d$ symmetry. Even in 
presumably simple metals, these unoccupied $d$ states approach the Fermi level as $Z$ increases and they can be probed via dipole-forbidden $s \rightarrow d$ transitions due to a finite exchanged wave vector. ${ }^{48,49}$ Thus, the current understanding is that the prominent fine structure in the dielectric response of many materials at larger $Q$ is mainly due to band structure effects and can be modeled with $a b$ initio methods such as time-dependent density-functional theory (TDDFT) for metals, ${ }^{49-51}$ transition metals, ${ }^{52}$ and semiconductors. ${ }^{53-55}$ In this light, it has become a relevant question if there is at all a solid metal that exhibits a valence-electron dynamic structure factor representative for the HEG. To shed light on this question, we have chosen to study the dynamic structure factor of the Na valence electrons, since they are commonly considered one of the nature's closest realizations of a free-electron gas. In view of the importance of $\mathrm{Na}$ as such a model system with relatively weak electron-ion interaction, it is surprising that apart from the spectrum shown by Hill et al. ${ }^{56}$ no IXS measurements have been published to date, and that, in particular, no $a b$ initio theoretical spectra have been published at all.

In the present article, we study the dynamic structure factor of the valence electrons in polycrystalline sodium in the $Q$ range $0.5 k_{F}<Q<2.4 k_{F}$ using experimental IXS data. We compare the experimental results to those from calculations based on time-dependent density-functional theory within different levels of exchange-correlation approximations. ${ }^{57}$ In earlier studies, the plasmon dispersion in $\mathrm{Na}$ has been studied by EELS (Ref. 58) and analyzed theoretically ${ }^{51}$ within TDDFT using RPA and TDLDA but only in the low- $Q$ region $Q \lesssim k_{F}$. In those studies, it was found that while TDLDA explains the plasmon dispersion curves very well in the case of $\mathrm{Al}$, the agreement for $\mathrm{Na}$ is poor. This was attributed to exchange-correlation effects beyond the TDLDA. ${ }^{51}$ Moreover, it has been suggested that core polarization effects may play a role explaining the discrepancies. ${ }^{51}$ In the present work, we observe the transition from the collective-excitation regime to the single-particle domain and we show that correlations beyond the RPA - especially lifetime effectshave a large impact on the dynamic response function. The consideration of such lifetime effects allows us to obtain a good description of $S(Q, \omega)$ using TDLDA similarly to what has been obtained earlier in the case of $S(\mathbf{Q}, \omega)$ of silicon. ${ }^{54,55}$ By contrast, band structure effects turn out to be negligible in $\mathrm{Na}$, which is manifested by the lack of fine structure in the shape of the $S(Q, \omega)$ at larger momentum transfers.

TABLE I. The relevant constants for Na and HEG at the same density. The calculations have been performed using the constants at $T=300 \mathrm{~K}$. The $Q_{c}$ value can only be given approximatively.

\begin{tabular}{llllll}
\hline \hline & \multicolumn{3}{c}{$\mathrm{Na}$} & & \multicolumn{2}{c}{$\mathrm{HEG}$} \\
\cline { 2 - 3 } \cline { 5 - 6 } Quantity & $6 \mathrm{~K}$ & $300 \mathrm{~K}$ & & $6 \mathrm{~K}$ & $300 \mathrm{~K}$ \\
\hline$r_{s}$ & 3.93 & 3.99 & & 3.93 & 3.99 \\
$k_{F} a_{0}$ & 0.487 & 0.480 & & 0.488 & 0.481 \\
$\varepsilon_{F}(\mathrm{eV})$ & 3.227 & 3.135 & & 3.240 & 3.148 \\
$Q_{c} a_{0}$ & \multicolumn{3}{c}{$\sim 0.4$} \\
\hline \hline
\end{tabular}

The article is arranged as follows. The experimental and numerical methods are outlined in Sec. II. Results are presented and discussed in Sec. III. Conclusions are drawn in Sec. IV. The constants used in the calculations for $\mathrm{Na}$ and HEG are given in Table I.

\section{METHODS}

\section{A. Experiment}

The experiments were performed at the beamline ID16 of the European Synchrotron Radiation Facility. The incident photon beam was monochromated using a combination of a liquid-nitrogen-cooled double-crystal $\mathrm{Si}(111)$ premonochromator and a $\mathrm{Si}(220)$ channel-cut into a bandwidth of $0.5 \mathrm{eV}$ at $9.7 \mathrm{keV}$. The spectrometer is described in detail in Ref. 59. It is designed for studies of electronic excitations using the nonresonant IXS technique. We used two analyzer crystals, which were bent $\mathrm{Si}(110)$ wafers (bending radius $R=1 \mathrm{~m}$, wafer diameter $100 \mathrm{~mm}$ ) using the $\mathrm{Si}(660)$ reflection order with a fixed Bragg angle of $\theta_{B} \approx 89^{\circ}$. The measurements were done in the so-called inverse geometry where the incident-photon energy is scanned and the scattering intensity into a fixed scattering angle with a fixed photon energy is observed. The incident-photon energies were tuned between 9.7 and $9.8 \mathrm{keV}$ to reach energy transfers of $0-100 \mathrm{eV}$. The studied momentum transfer range was $Q=0.18-1.16 a_{0}^{-1}$, i.e., up to the regime where the plasmon enters the particle-hole continuum. The energy resolution was $1.0 \mathrm{eV}$ and the momentum-transfer resolution was $0.05 a_{0}^{-1}$.

The sample was prepared in a helium-flow cryostat in a glove box filled with argon and transported to the beamline within the argon atmosphere. At the beamline, the cryostat was evacuated to a vacuum of $10^{-6}$ mbar. The sample was polycrystalline to a good approximation. No visible degradation of the sample surface was observable throughout the experiment. The spectra were measured both at 300 and $6 \mathrm{~K}$ to determine possible thermal effects on the spectra. The Debye temperature of $\mathrm{Na}$ is $160 \mathrm{~K}$ and the melting temperature is $371 \mathrm{~K}$, so measurements at room temperature could be affected by thermal vibrations of the ion core lattice. ${ }^{60}$

All spectra were measured several times in order to determine any time-dependent changes in the sample or instabilities. None was found, and the individual spectra were averaged after normalization to the incident photon flux. The zero-energy loss peak had a significant contribution to the spectra in the energy region $\omega<3 \mathrm{eV}$. It was removed from the spectra, for visualization purposes, by replacing data in that region by a polynomial fit to the low-energy tail of the $S(Q, \omega)$, keeping $S(Q, 0)=0$ and $d S(Q, \omega) /\left.d \omega\right|_{\omega=0}=0$. Thus, our experimental spectra in the region $\omega<3 \mathrm{eV}$ should not be taken as quantitatively reliable. Due to the overlap of the signal of the $\mathrm{Na} L$ electrons with the high-energy tail of the valence $S(Q, \omega)$, the use of the $f$-sum rule ${ }^{1}$ was not possible to bring the spectra to an absolute $S(Q, \omega)$ scale. Thus, the experimental spectra were normalized to the same area as the theoretical ones within the lifetime approximation (see below) in the energy range 5-25 eV. This procedure, which has been discussed in details in Ref. 55, is reliable due to the good agreement of the spectral shapes. 


\section{B. Theory and computational details}

The calculations were done in the framework of linearresponse time-dependent density-functional theory. ${ }^{61,62} \mathrm{Ap}-$ proximate finite lifetimes of electrons and holes were included via a modified independent-particle polarizability $\chi_{0}^{\mathrm{LT}}$, where the imaginary part of the self-energy (which represents the lifetimes of the electrons and holes independently) appears in the denominator.

This approach, described in detail in Refs. 55 and 57, is based on the observation that, in principle, the TDDFT kernel, which has to simulate a full many-body result, should contain two contributions: a term originating from the difference between the Kohn-Sham theory and the fully interacting one-body Green's function $G$, and a term for the electron-hole interaction in the Bethe-Salpeter equation. ${ }^{63}$ In the BetheSalpeter framework, the most common approximations are: (i) to replace the fully interacting one-body Green's function $G$ by a quasiparticle Green's function and (ii) to replace the electron-hole interaction kernel by a statically screened Coulomb interaction $W$. Such an approximation is justified because dynamical effects in $G$ and $W$ are known to partially cancel each other (see, e.g., Refs. 64 and 65). Indeed, most often results are excellent. Since the static $W$ is real, one can immediately see that damping in the final result stems from finite lifetimes in $G$ only. Such a Bethe-Salpeter calculation has been performed, e.g., for the EELS spectrum of silicon for vanishing momentum transfer, ${ }^{66}$ showing that the inclusion of the imaginary part of the quasiparticle energies in the calculation leads to a significant improvement with respect to a calculation supposing infinite lifetimes of electrons and holes. The real part of the self-energy corrections, instead, leads in to a shift of the energy levels with respect to a Kohn-Sham result. If one supposes that the main effect is a rigid shift of the bands, with no changes in the wave functions, one finds a cancellation between the self-energy corrections to the gap and electron-hole interaction effects beyond the (time-dependent) Kohn-Sham theory. In many materials, and certainly in silicon, the hypothesis of a rigid self-energy correction together with an absence of change in the wave functions with respect to the LDA wave functions is reasonable, and indeed strong cancellations between these contributions are observed. This is particularly true for loss spectra (see, e.g., Ref. 67), where the energy scale is much larger than the error bar of this approximation. The conclusion is then that the effects of the real part of the self-energy can be replaced by the TDLDA kernel because of cancellations, and that the effects of the imaginary part, that show up in the independent quasiparticle response only, simply require to add this imaginary part to the corresponding energies. By doing this, TDLDA+LT calculations have been shown to lead to a very good agreement with the experiments, except for very large momentum transfer $\left(Q>2 k_{F}\right)$, where the agreement is slightly less good. ${ }^{54,55}$

All the calculations are done using the plane-wave code ABINIT (Ref. 68) for the ground-state calculation, and the DP $\operatorname{code}^{69}$ for the linear-response calculation. The electron-ion interaction is described using norm-conserving pseudopotentials. The calculations have been converged with respect to all computational parameters. In particular, a $16 \times 16 \times$

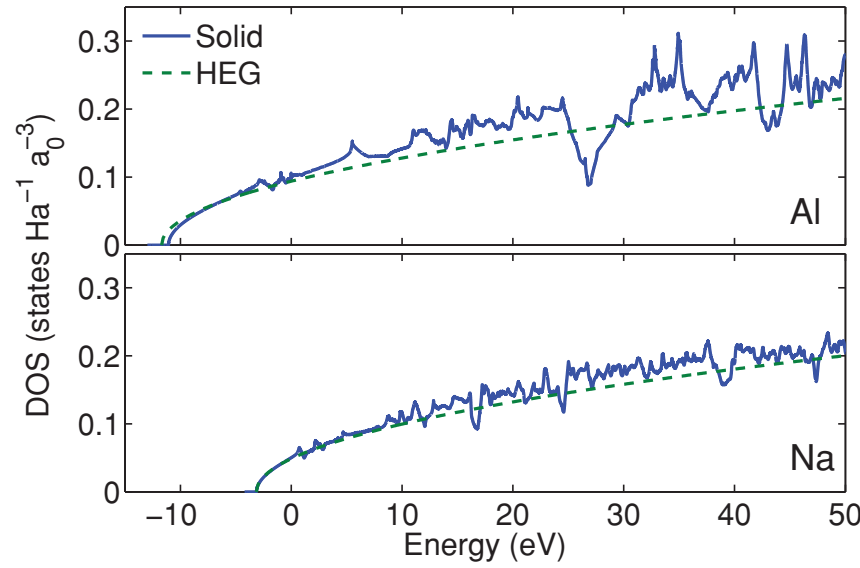

FIG. 1. (Color online) Electron DOS for aluminum and sodium as calculated within the LDA and compared to the corresponding DOS for HEG at equal densities. The Fermi energies are here set to $\varepsilon_{F}=0$.

16 shifted $k$-point mesh has been used and the electronic temperature was set between $5 \times 10^{-5}$ and $5 \times 10^{-4}$ hartree, depending on $Q$.

\section{RESULTS AND DISCUSSION}

As discussed in Sec. I, all earlier IXS measurements of simple metals have revealed pronounced fine structure in the $S(Q, \omega)$ for $Q>Q_{c}$, where the measurement probes electronhole pair excitations. This can usually be traced back to the fine structure of the single-particle DOS, which even in metals that are often considered HEG-like, shows deviations from the HEG behavior above the Fermi energy. This is exemplified in our calculated DOS for $\mathrm{Al}$ and $\mathrm{Na}$ in Fig. 1. The large gap that is clearly visible in $\mathrm{Al}$ at around $28 \mathrm{eV}$ causes the dip in its dynamic response, reported, e.g., in Ref. 48. The unoccupied DOS of Na, on the other hand, is closer to being free-electron-like. Thus, we would expect less lattice-induced fine structure in the $S(Q, \omega)$ of Na than in Al. However, even for $\mathrm{Na}$, the DOS above $\varepsilon_{F}$ does show some nonsmooth structure, so even $\mathrm{Na}$ could be expected to show small fine structure based on the above-mentioned argument. Indeed, some effects on lattice-induced structures in the spectra of $\mathrm{Na}$ appear in the experiments of Refs. 58 and 70.

The measured dynamic structure factors of Na at $T=300 \mathrm{~K}$ are compared to the calculations at four different levels of theory in Fig. 2. The plasmon in the HEG RPA model has an energy $\omega_{\mathrm{pl}}(Q)=\omega_{0}+\alpha Q^{2}+O\left(Q^{4}\right)$, where $\alpha$ is the plasmon dispersion constant. The spectra at $Q<Q_{c}$ are dominated by the plasmon, which at $Q=0$ has the energy $\omega_{0}=5.9 \mathrm{eV} .^{58}$ The spectra become broader and the peak continues to shift toward higher energies with increasing $Q$, as it is known for the HEG response.

Now, the most interesting result seen here is that the measured $S(Q, \omega)$ shows no fine structure for $Q>2 Q_{c}$, in clear contrast to practically any other material studied by IXS. ${ }^{5}$ However, both the RPA and the TDLDA results do show a fine structure starting at $Q \approx 1.5 k_{F}$, in a form of a dip-shoulder structure at around $15 \mathrm{eV}$ energy transfer. This structure is washed out in the TDLDA+LT calculation. The small excitation gaps visible in the DOS of Fig. 1 produce these 

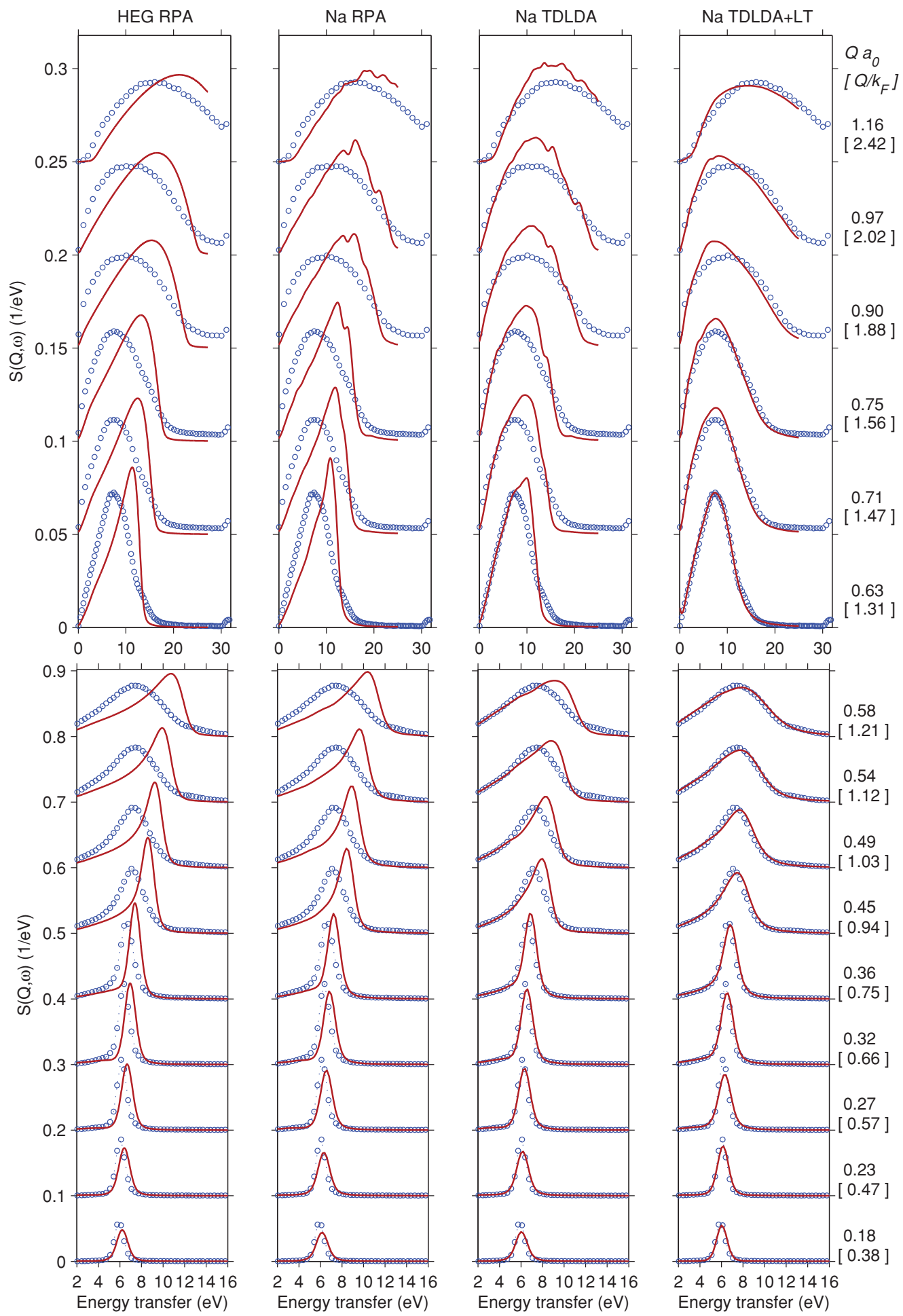

FIG. 2. (Color online) Experimental $S(Q, \omega)$ at $T=300 \mathrm{~K}$ (circles) compared to calculations (lines) for the HEG within RPA, and for real sodium metal within RPA, TDLDA, and TDLDA+LT for selected momentum transfers $Q$. The spectra are shifted vertically in steps of $0.11 / \mathrm{eV}$ (lower panel), and $0.051 / \mathrm{eV}$ (upper panel). The momentum-transfer values for each spectrum are indicated on the right both in units of $a_{0}^{-1}$ and in units of the Fermi wave vector $k_{F}$ (latter in brackets).

structures in RPA and TDLDA, but the finite lifetime effects smoothen the spectrum, completely removing any dip-like fine structure.

The good performance of this modified TDLDA approach is noteworthy, especially in the view of its simplicity and the corresponding shortcomings. At small values of $Q$, the TDLDA kernel has little influence because it is independent of $Q$; hence it misses the $Q^{-2}$ divergence of the Coulomb interaction, which leads to problems in describing optical spectra. As soon as $Q$ increases, the relative weight of $f_{\mathrm{xc}}^{\mathrm{TDLDA}}$ 
with respect to the RPA (Coulomb) contribution $v_{C}=4 \pi / Q^{2}$ becomes larger, and starts to influence the resulting $S(Q, \omega)$. TDLDA, in fact, yields a better agreement with the experiment than RPA. However, it should be noted that the TDLDA $f_{\mathrm{xc}}^{\text {TDLDA }}$ is also incorrect in the large- $Q$ limit, where problems are expected since the sum of $f_{\mathrm{xc}}^{\mathrm{TDLDA}}$ (a negative constant) and the Coulomb term $v_{C}$ changes sign. At sufficiently large values of $Q$, this problem will show up, as observed in several works (e.g., in Refs. 71 and 72). However, no unphysical behavior in $S(Q, \omega)$ has been observed in literature up to momentum transfers of $2.98 \AA^{-1}$ for Sc, $3.5 \AA^{-1}$ for $\mathrm{Cr},{ }^{52}$ or $1.7 \AA^{-1}$ for diamond. ${ }^{8}$ Indeed, our findings here are similar to those obtained for bulk silicon, where TDLDA likewise improves distinctly over RPA. ${ }^{55}$

The trend of both theoretical and experimental results can be very clearly seen in Fig. 2: the RPA typically continues with the low- $Q$ plasmon dispersion relation even at finite $Q$, giving a rather sharp excitation, with the peak position at clearly higher energies than seen in the experiment. The tendency of short-range correlations, not included in the RPA, is above all to slow down the excitation-spectrum peak dispersion when the collective excitations start to decay into particle-hole excitations (which are of the short-range type). This is evidenced by the more accurate description given by the TDLDA as compared to that of the RPA. An apparent consequence to the retarded dispersion is a simultaneous broadening of the spectra. In other words, the particle-hole excitation regime seems to have stronger effect on the experimental $S(Q, \omega)$ than predicted by the RPA. This is a consequence of short-range interactions that tend to bring the electron-gas behavior toward a single-particle (i.e., Compton) response, but which are not included in the RPA. However, even TDLDA is not sufficient to fully predict the experimental spectra. Much better agreement is obtained by an additional inclusion of quasiparticle lifetimes ${ }^{55,57}$ with the TDLDA+LT treatment.

There are only two distinct features in the experimental spectra that are not described by the theoretical TDLDA+LT results. First, the jump in the experimental data at $\sim 31 \mathrm{eV}$ is due to the $\mathrm{Na} L$ edge; with energy transfer values above this value, the excitation of $\mathrm{Na} 2 p$ electrons to the unoccupied states is possible. This feature is not present in the theory, because in the calculations the $2 p$ electrons were included in the frozen pseudopotential core. The spectra at this region correspond very closely to those of soft x-ray absorption spectroscopy (XAS) of the corresponding electrons. This branch of IXS is called X-ray Raman spectroscopy (XRS) and is an increasingly important tool that complements XAS experiments. The $\mathrm{Na} L$ edges have been studied in detail via XRS experimentally ${ }^{73}$ and theoretically. ${ }^{74}$ Second, the spectra show the so-called double-plasmon excitation peak, which is a signature of creation of intrinsic double plasmons in the excitation process, ${ }^{75,76}$ and which can be seen at intermediate $Q$ values at $12-14 \mathrm{eV}$, i.e., at roughly twice the plasmon energy. This peak is understood to be a consequence of higher level correlations, ${ }^{77}$ not taken into account in any of our theoretical approaches used here, and correspondingly, not seen in any of the theoretical curves.

In Fig. 3, we present the experimental and theoretical dispersion of the $S(Q, \omega)$ peak position, superimposed with $S(Q, \omega)$ contour plots for $\mathrm{Na}$ at both $6 \mathrm{~K}$ [Fig. 3(a)] and $300 \mathrm{~K}$
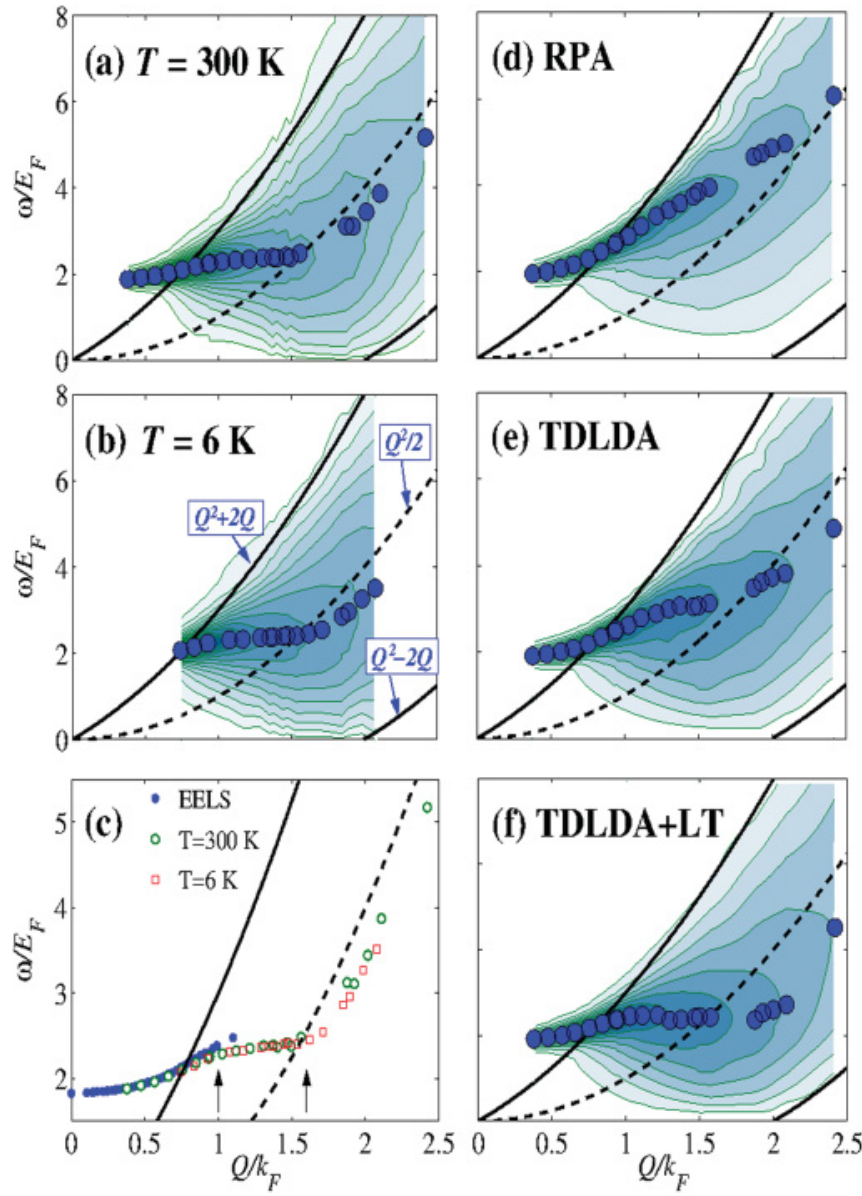

FIG. 3. (Color online) (a) and (b) The dynamic structure factor for $\mathrm{Na}$ and the dispersion of the energy of its peak from experimental data in different temperatures. The particle-hole continuum is marked with lines and the single-particle excitation dispersion $Q^{2} / 2 m$ with the dashed line, as annotated in (b). (c) Both experimental dispersions overlaid with results from electron-energy-loss spectroscopy. ${ }^{58}$ Note the different ordinate axis in (c). Arrows mark the kinks in the dispersion; first a flattening at $Q \approx k_{F}$ and an acceleration at $Q \approx 1.6 k_{F}$. (d)-(f) Theoretical dispersion contours. We assume here $m^{*}=m=1$.

[Fig. 3(b)]. The figures also show the location of the customary particle-hole continuum assuming an effective mass $m^{*}=m_{e}$. It has been shown that for elemental metals, in this density range, this approximation is quite good, ${ }^{78}$ with the values $m^{*} / m_{e}$ varying between 0.953 and 1.068 . The figures also show the Compton recoil energy $Q^{2} / 2 m^{*}$ which corresponds to the limit of scattering from a single particle in the impulse approximation. The $S(Q, \omega)$ peak position will meet this curve at large $Q$. What exactly happens when the response changes from the plasmon limit at $0<Q<Q_{c}$ to the Compton limit $Q \gg Q_{c}$ is not trivial. The experimental peak positions are found to first follow a positive dispersion, i.e., an increase of the peak-position energy with increasing $Q$, but then a strong flattening takes place between $k_{F}<Q<2 k_{F}$. In this $Q$ region, the plasmon has very little dispersion. There is a crossing of the single-particle limit for the experimental $S(Q, \omega)$ peak position around $1.5 k_{F}$, after which the dispersion sets in again, becoming very strong while getting closer to 
the $E=Q^{2} / 2$ limit at our highest measured $Q$. In Fig. 3(c), our IXS results are plotted in comparison with earlier EELS results. ${ }^{58}$ The agreement between IXS and EELS results is very good. Exactly at the highest $Q$ of the EELS measurements (at $Q \sim k_{F}$ ), the above-mentioned dispersion-flattening takes place as seen in our IXS results. A very similar leveling-off behavior is seen in the normal liquid ${ }^{3} \mathrm{He}$ (Ref. 79), where, however, the particle-hole continuum is upshifted in both $Q$ and $\omega$ due to the large effective mass $m^{*} \approx 3 m$. This may mean that the overall shape of the dispersion relation could be universal to the Fermi gas. As discussed above, the reasons for the dispersion behavior may be due to short-range correlations not taken into account in the RPA. The onset of the short-range interactions takes place at around $k_{F}$, after which the single-particle continuum plays a larger role in the dynamic response of the electron gas than is described by the RPA.

For the theoretical peak dispersion, the RPA result follows closely the usual $E=E_{0}+\alpha Q^{2}+O\left(Q^{4}\right)$ behavior until $\sim Q_{c}$, thereafter slowing down in the particle-hole continuum and meeting the single-particle limit at around $2.3 k_{F}$. The TDLDA brings the theory to a better agreement with the experiment, with an effect of an increased slowing down of the dispersion, and even bending down below the single-particle limit at $1.7 k_{F}$. However, it still shows some discrepancies with respect to the experimental results. On the other hand, after the additional inclusion of the quasiparticle lifetime effects in the TDLDA+LT calculations, the agreement with the experiment is very good. It is noteworthy that the kernel has such a large effect on the spectra and that both RPA and TDLDA fail to give a correct description of the dispersion. Moreover, the quasiparticle lifetimes play an important role in the dynamic response of the system. The implications of this and the details on the TDLDA+LT approximation are published in a separate paper. ${ }^{57}$ We mention that in the inclusion of the lifetime effects, the true lifetimes of sodium have to be used. Approximating them with those of the HEG leads to a double-peak structure not found in the experiment. ${ }^{57}$

It is interesting to compare our results with earlier theoretical investigations by Quong and Eguiluz, ${ }^{51}$ who studied the dispersion relation in $\mathrm{Al}$ and $\mathrm{Na}$ taking all band-structure effects into account, and treating the exchange-correlation problem at the TDLDA level. They showed that in Al, the plasmon dispersion is largely affected by lattice effects, whereas this is much less the case in Na. Already their analysis showed that TDLDA, while working well in $\mathrm{Al}$, fails to give the correct dispersion behavior in $\mathrm{Na}$. However, since the experimental data available at that time accessed only the $Q<k_{F}$ region, the complete description of the dispersion flattening was not thoroughly discussed.

As for earlier experimental results, it is useful to discuss especially those of Larson et al. ${ }^{80}$ and Schülke et al. ${ }^{81}$. In the spirit of the earlier many-particle discussions on the HEG model, it has been customary to refer to the socalled local-field correction $G(\mathbf{Q}, \omega)$ which, multiplied by the Coulomb potential $v_{C}(Q)$, is equivalent to the exchangecorrelation kernel $f_{\mathrm{xc}}(\mathbf{Q}, \omega)$ of TDDFT. In both cases, setting $-v_{C}(Q) G(\mathbf{Q}, \omega)=f_{\mathrm{xc}}(\mathbf{Q}, \omega)=0$ corresponds to the RPA. $G(Q, \omega)$ describes the difference between the RPA prediction and the exact response in such a way that if $\operatorname{Re} G(Q, \omega)=1$, the exact response is that of noninteracting electrons (and the excitation dispersion follows the dashed line in Fig. 3). At $Q=1.5 k_{F}$, at least the dispersion of the experimental $S(Q, \omega)$ maximum shows exactly this behavior. When the exact result crosses the $Q^{2} / 2 m^{*}$ curve, then $\operatorname{Re} G(Q, \omega)>1$. This happens in our experimental data in the region $1.5 k_{F}<Q \lesssim 2.5 k_{F}$. The static approximation of $G(Q, \omega)$ corresponds to neglecting the $\omega$ dependence, and such static local-field factors were derived experimentally for $\mathrm{Li}$ (Ref. 81) and for Al. ${ }^{80}$ Those studies found out exactly the above-described behavior for the $\operatorname{Re} G(Q)$ with a crossing point at $Q=1.5 k_{F}$, a value that seems to be the same in $\mathrm{Li}, \mathrm{Al}$, and now also in $\mathrm{Na}$.

Sternemann et al. ${ }^{60}$ studied the temperature dependence of the dynamic structure factor of aluminum and showed that the prominent double-peak structure was diminished by an increase of temperature up to the melting point. This was explained to be due to the temperature dependence of the Debye-Waller factor which causes a smearing of the lattice-induced fine structure when the lattice starts to be less well defined at higher temperatures. As mentioned earlier, the Debye temperature of $\mathrm{Na}$ is $160 \mathrm{~K}$ and the melting temperature is $371 \mathrm{~K}$, so measurements at room temperature could be significantly affected by temperature effects on the lattice, if the electron-ion-lattice interaction had such a strong influence on the spectra for $\mathrm{Na}$. To study this, we performed the experiment at $6 \mathrm{~K}$ and at room temperature. However, within the experimental accuracy, no temperature dependence was found except for the trivial density dependence of the plasmon energy. Possible explanations for the lack of any evident temperature dependence may be that the latticeinduced contributions to $S(Q, \omega)$ are very small, or, if they exist, that their temperature dependence is small.

\section{CONCLUSIONS}

We have measured the valence dynamic response of sodium and compared the results with TDDFT calculations. The almost complete absence of fine structure in the $S(Q, \omega)$ shape in Na clearly corroborates the fact that the previously observed double-peak or peak-shoulder structure in other simple metals has its origin in band-structure effects. This is known from previous studies where the spectral structures have been found to depend strongly on the crystal orientation, as well as on the temperature $^{60}$ that affects the electron-lattice interaction via a softening of the lattice potential (i.e., thermal disorder). By contrast, the $\mathrm{Na}$ data without fine structure shown here give hints toward what the HEG $S(Q, \omega)$ could look like.

The dispersion of the $S(Q, \omega)$ peak was found to slow down considerably compared to the RPA prediction, leading to a flattening of the dispersion in the region $k_{F}<Q<2 k_{F}$. The peak dispersion (and the lineshape) meets the single-particle dispersion limit $E=Q^{2} / 2$ at $Q \approx 1.5 k_{F}$, after which it stays below this value up to our largest measured $Q \approx 2.5 k_{F}$, where the experimental dispersion meets the single-particle limit again. This is possibly due to a relatively important contribution of short-range correlation in this range, promoting the decay of the plasmon into single-particle excitations more efficiently than described in the RPA. The dispersion curve bears similarities to those of normal liquid ${ }^{3} \mathrm{He}$ (Ref. 79) 
suggesting that the overall shape of the dispersion relation could be universal to a Fermi gas.

Comparison of the experimental results with theoretical predictions within TDDFT suggests that the effect of correlation is rather large in the case of $\mathrm{Na}$, evidenced also by the rather pronounced difference between the theoretical RPA and TDLDA results. TDLDA provides better agreement with the experiment, although quite substantial differences remain. This remaining discrepancy is corrected when additionally the effects of quasiparticle lifetimes are taken into account in the TDLDA+LT approximation. As discussed in detail elsewhere ${ }^{57}$ the inclusion of the lifetimes has to be based on the quasiparticle lifetimes of the real Na metal, while the use of the lifetimes calculated for the HEG produces a shoulder-type fine structure not present in the experimental result. Thus, even the data on the dielectric response shown here are not fully representative of the HEG response. However, in contrast to results of other studies on simple metals and semiconductors, the deviation from the HEG response in Na does not come from the effect of the electron-ion interaction on the band structure but rather from its effect on the lifetimes. ${ }^{57}$

Therefore, we are finally in the position to offer an answer to the question whether the valence-electron dynamics in $\mathrm{Na}$ can be regarded as being HEG-like to a good approximation. Our answer is both yes and no. The quasiparticle lifetimes wash out the small bandstructure effects visible in both the RPA and the TDLDA spectra. However, the electron-ion interaction must be taken into account in the calculation of the lifetimes in order to avoid the introduction of a spurious double-peak structure.

\section{ACKNOWLEDGMENTS}

The authors would like to thank N. Manini, V. Nazarov, G. Vignale, A. Marini, R. Del Sole, V. Olevano, and F. Sottile for enlightening discussions and support. The work was supported by the European Union through the ETSF e-I3 Grant No. INFRA-2007-211956. Beam time was provided by the European Synchrotron Radiation Facility. The computations were done using HPC resources from GENCI-IDRIS (Grant No. 2009-544), as well as from CINECA through the Calcolo per la Fisica della Materia initiative. H.-Ch.W. acknowledges support from the European Union through the individual Marie Curie Intra-European Grant No. MEIF-CT-2005-025067, as well as from the Physics Department of the Ecole Polytechnique, Palaiseau, France. T.P. was supported by the Academy of Finland via the Contract No. 1127462. S.H. was supported by Research Funds of the University of Helsinki, Contract No. 490076. *simo.huotari@helsinki.fi

${ }^{\dagger}$ The $\mathrm{CINaM}$ is associated to the Universities of Aix-Marseille II and III.

${ }^{1}$ D. Pines and P. Noziéres, The Theory of Quantum Liquids (Benjamin, New York, 1966), Vol. 1.

${ }^{2}$ J. Fink, Adv. Electron. Electron Phys. 75, 121 (1989).

${ }^{3}$ F. J. García de Abajo, Rev. Mod. Phys. 82, 209 (2010).

${ }^{4}$ W. Schülke, J. Phys. Condens. Matter 13, 7557 (2001).

${ }^{5}$ W. Schülke, Electron Dynamics by Inelastic X-Ray Scattering (Oxford University Press, Oxford, 2007).

${ }^{6} X$-Ray Compton Scattering, edited by M. J. Cooper, P. E. Mijnarends, N. Shiotani, N. Sakai, and A. Bansil, (Oxford University Press, Oxford, 2004).

${ }^{7}$ S. Huotari et al., Phys. Rev. Lett. 105, 086403 (2010).

${ }^{8}$ S. Waidmann, M. Knupfer, B. Arnold, J. Fink, A. Fleszar, and W. Hanke, Phys. Rev. B 61, 10149 (2000).

${ }^{9}$ C. A. Burns, P. Abbamonte, E. D. Isaacs, and P. M. Platzman, Phys. Rev. Lett. 83, 2390 (1999).

${ }^{10}$ C. A. Burns, P. Giura, A. Said, A. Shukla, G. Vankó, M. TuelBenckendorf, E. D. Isaacs, and P. M. Platzman, Phys. Rev. Lett. 89, 236404 (2002).

${ }^{11}$ G. Tirao, G. Stutz, V. M. Silkin, E. V. Chulkov, and C. Cusatis, J. Phys. Condens. Matter 19, 046207 (2007).

${ }^{12}$ U. Bergmann and S. P. Cramer, Proc. SPIE 3448, 198 (1998).

${ }^{13}$ Y. Q. Cai, P. Chow, C. C. Chen, H. Ishii, K. L. Tsang, C. C. Kao, K. S. Liang, and C. T. Chen, AIP Conf. Proc. 705, 340 (2004).

${ }^{14}$ T. T. Fister, G. T. Seidler, L. Wharton, A. R. Battle, T. B. Ellis, J. O. Cross, A. T. Macrander, W. T. Elam, T. A. Tyson, and Q. Qian, Rev. Sci. Instrum. 77, 063901 (2006).
${ }^{15}$ J. P. Hill, D. S. Coburn, Y.-J. Kim, T. Gog, D. M. Casa, C. N. Kodituwakku, and H. Sinn, J. Synchrotron Radiat. 14, 361 (2007).

${ }^{16}$ J.-L. Hazemann et al., J. Synchrotron Radiat. 16, 283 (2009).

${ }^{17}$ E. Collart, A. Shukla, F. Gélébart, M. Morand, C. Malgrange, N. Bardou, A. Madouri, and J.-L. Pelouard, J. Synchrotron Radiat. 12, 473 (2005)

${ }^{18}$ S. Huotari, G. Vankó, F. Albergamo, C. Ponchut, H. Graafsma, C. Henriquet, R. Verbeni, and G. Monaco, J. Synchrotron Radiat. 12, 467 (2005).

${ }^{19}$ R. Verbeni, M. Kocsis, S. Huotari, M. Krisch, G. Monaco, F. Sette, and G. Vankó, J. Phys. Chem. Solids 66, 2299 (2005).

${ }^{20}$ P. Eisenberger, P. M. Platzman, and K. C. Pandy, Phys. Rev. Lett. 31, 311 (1973).

${ }^{21}$ P. M. Platzman and P. Eisenberger, Phys. Rev. Lett. 33, 152 (1974).

${ }^{22}$ P. Eisenberger, P. M. Platzman, and P. Schmidt, Phys. Rev. Lett. 34, 18 (1975).

${ }^{23}$ G. Mukhopadhyay, R. K. Kalia, and K. S. Singwi, Phys. Rev. Lett. 34, 950 (1975).

${ }^{24}$ H. De Raedt and B. De Raedt, Phys. Rev. B 18, 2039 (1978).

${ }^{25}$ F. Yoshida, S. Takeno, and H. Yasuhara, Prog. Theor. Phys. 64, 40 (1980).

${ }^{26}$ K. Awa, H. Yasuhara, and T. Asahi, Solid State Commun. 38, 1285 (1981).

${ }^{27}$ K. Awa, H. Yasuhara, and T. Asahi, Phys. Rev. B 25, 3670 (1982).

${ }^{28}$ K. Awa, H. Yasuhara, and T. Asahi, Phys. Rev. B 25, 3687 (1982).

${ }^{29}$ F. Green, D. N. Lowy, and J. Szymański, Phys. Rev. Lett. 48, 638 (1982).

${ }^{30}$ G. Niklasson, A. Sjölander, and F. Yoshida, J. Phys. Soc. Jpn. 52, 2140 (1983). 
${ }^{31}$ S. Rahman and G. Vignale, Phys. Rev. B 30, 6951 (1984).

${ }^{32}$ F. Green, D. Neilson, and J. Szymański, Phys. Rev. B 31, 5837 (1985).

${ }^{33}$ J. Hong and M. H. Lee, Phys. Rev. Lett. 55, 2375 (1985).

${ }^{34}$ G. Mukhopadyay and A. Sjölander, Phys. Rev. B 17, 3589 (1978).

${ }^{35}$ K. Bedell and G. E. Brown, Phys. Rev. B 17, 4512 (1978).

${ }^{36}$ H. Hayashi and M. Shimizu, J. Phys. Soc. Jpn. 48, 16 (1980).

${ }^{37}$ E. Pajanne, J. Phys. C 15, 5629 (1982).

${ }^{38}$ N. Iwamoto, E. Krotscheck, and D. Pines, Phys. Rev. B 29, 3936 (1984).

${ }^{39}$ A. Holas and S. Rahman, Phys. Rev. B 35, 2720 (1987).

${ }^{40}$ G. Mukhopadhyay, Phys. Scr. 38, 224 (1988).

${ }^{41}$ J. Hong and M. H. Lee, Phys. Rev. Lett. 70, 1972 (1993).

${ }^{42}$ M. E. Bachlechner, A. Holas, H. M. Böhm, and A. Schinner, Phys. Rev. B 54, 2360 (1996).

${ }^{43}$ Y. Takada and H. Yasuhara, Phys. Rev. Lett. 89, 216402 (2002).

${ }^{44}$ W. Schülke, H. Nagasawa, and S. Mourikis, Phys. Rev. Lett. 52, 2065 (1984).

${ }^{45}$ W. Schülke, H. Nagasawa, S. Mourikis, and P. Lanzki, Phys. Rev. B 33, 6744 (1986).

${ }^{46}$ W. Schülke, U. Bonse, H. Nagasawa, S. Mourikis, and A. Kaprolat, Phys. Rev. Lett. 59, 1361 (1987).

${ }^{47}$ W. Schülke, H. Nagasawa, S. Mourikis, and A. Kaprolat, Phys. Rev. B 40, 12215 (1989).

${ }^{48}$ W. Schülke, H. Schulte-Schrepping, and J. R. Schmitz, Phys. Rev. B 47, 12426 (1993).

${ }^{49}$ S. Huotari, C. Sternemann, M. C. Troparevsky, A. G. Eguiluz, M. Volmer, H. Sternemann, H. Müller, G. Monaco, and W. Schülke, Phys. Rev. B 80, 155107 (2009).

${ }^{50}$ A. Fleszar, A. A. Quong, and A. G. Eguiluz, Phys. Rev. Lett. 74, 590 (1995).

${ }^{51}$ A. A. Quong and A. G. Eguiluz, Phys. Rev. Lett. 70, 3955 (1993).

${ }^{52}$ I. G. Gurtubay, J. M. Pitarke, W. Ku, A. G. Eguiluz, B. C. Larson, J. Tischler, P. Zschack, and K. D. Finkelstein, Phys. Rev. B 72, 125117 (2005).

${ }^{53}$ W. Schülke, J. R. Schmitz, H. Schulte-Schrepping, and A. Kaprolat, Phys. Rev. B 52, 11721 (1995).

${ }^{54}$ H.-Ch. Weissker, J. Serrano, S. Huotari, F. Bruneval, F. Sottile, G. Monaco, M. Krisch, V. Olevano, and L. Reining, Phys. Rev. Lett. 97, 237602 (2006).

${ }^{55}$ H.-Ch. Weissker, J. Serrano, S. Huotari, E. Luppi, M. Cazzaniga, F. Bruneval, F. Sottile, G. Monaco, V. Olevano, and L. Reining, Phys. Rev. B 81, 085104 (2010).

${ }^{56}$ J. P. Hill, C.-C. Kao, W. A. C. Caliebe, D. Gibbs, and J. B. Hastings, Phys. Rev. Lett. 77, 3665 (1996).
${ }^{57}$ M. Cazzaniga, H.-Ch. Weissker, S. Huotari, T. Pylkkänen, P. Salvestrini, G. Monaco, G. Onida, and L. Reining, Phys. Rev. B 84, 075109 (2011).

${ }^{58}$ A. vom Felde, J. Sprösser-Prou, and J. Fink, Phys. Rev. B 40, 10181 (1989).

${ }^{59}$ R. Verbeni, T. Pylkkänen, S. Huotari, L. Simonelli, G. Vankó, K. Martel, C. Henriquet, and G. Monaco, J. Synchrotron Radiat. 16, 469 (2009).

${ }^{60}$ C. Sternemann, A. Kaprolat, and W. Schülke, Phys. Rev. B 57, 622 (1998).

${ }^{61}$ E. Runge and E. K. U. Gross, Phys. Rev. Lett. 52, 997 (1984).

${ }^{62}$ S. Botti, A. Schindlmayr, R. Del Sole, and L. Reining, Rep. Prog. Phys. 70, 357 (2007).

${ }^{63}$ F. Bruneval, F. Sottile, V. Olevano, R. Del Sole, and L. Reining, Phys. Rev. Lett. 94, 186402 (2005).

${ }^{64}$ F. Bechstedt, K. Tenelsen, B. Adolph, and R. Del Sole, Phys. Rev. Lett. 78, 1528 (1997).

${ }^{65}$ A. Marini and R. Del Sole, Phys. Rev. Lett. 91, 176402 (2003).

${ }^{66}$ B. Arnaud, S. Lebègue, and M. Alouani, Phys. Rev. B 71, 035308 (2005).

${ }^{67}$ V. Olevano and L. Reining, Phys. Rev. Lett. 86, 5962 (2001).

${ }^{68}$ The ABINIT code is a common project of the Université Catholique de Louvain, Corning Incorporated, and other contributors [http://www.abinit.org].

${ }^{69}$ V. Olevano et al., [http://www.dp-code.org].

${ }^{70}$ P. C. Gibbons and S. E. Schnatterly, Phys. Rev. B 15, 2420 (1977)

${ }^{71}$ J. F. Dobson and J. Wang, Phys. Rev. B 62, 10038 (2000).

${ }^{72}$ F. Furche and T. V. Voorhis, J. Chem. Phys. 122, 164106 (2005).

${ }^{73}$ C. Sternemann, J. A. Soininen, S. Huotari, G. Vankó, M. Volmer, R. A. Secco, J. S. Tse, and M. Tolan, Phys. Rev. B 72, 035104 (2005).

${ }^{74}$ H. Sternemann, J. A. Soininen, C. Sternemann, K. Hämäläinen, and M. Tolan, Phys. Rev. B 75, 075118 (2007).

${ }^{75}$ C. Sternemann, S. Huotari, G. Vankó, M. Volmer, G. Monaco, A. Gusarov, H. Lustfeld, K. Sturm, and W. Schülke, Phys. Rev. Lett. 95, 157401 (2005).

${ }^{76}$ S. Huotari, C. Sternemann, W. Schülke, K. Sturm, H. Lustfeld, H. Sternemann, M. Volmer, A. Gusarov, H. Müller, and G. Monaco, Phys. Rev. B 77, 195125 (2008).

${ }^{77}$ K. Sturm and A. Gusarov, Phys. Rev. B 62, 16474 (2000).

${ }^{78}$ G. E. Simion and G. F. Giuliani, Phys. Rev. B 77, 035131 (2008).

${ }^{79}$ F. Albergamo, R. Verbeni, S. Huotari, G. Vankó, and G. Monaco, Phys. Rev. Lett. 99, 205301 (2007).

${ }^{80}$ B. C. Larson, J. Z. Tischler, E. D. Isaacs, P. Zschack, A. Fleszar, and A. G. Eguiluz, Phys. Rev. Lett. 77, 1346 (1996).

${ }^{81}$ W. Schülke, K. Höppner, and A. Kaprolat, Phys. Rev. B 54, 17464 (1996). 